\title{
Phenotypic Plasticity and the Invasiveness of Three Taraxacum Species
}

\section{Jing Luo and John Cardina}

Department of Horticulture and Crop Science, The Ohio State University, Columbus, Ohio 43210. Email: luojess@gmail.com

\section{Introduction}

Phenotypic plasticity - the ability of a plant genotype to respond to different environmental conditions by producing different phenotypes is thought to play an important role in plant invasions.

Invasive species might benefit from phenotypic plasticity in three ways:

1) Jack-of-all-trades: invasive and non-invasive species have similar fitness in favorable environments, but invasive species have higher fitness in stressful environments (Fig. 1a)

2) Master-of-some: invasive and non-invasive species have similar fitness in stressful environments, but invasive species have higher fitness in favorable environments (Fig. 1b);

3) Jack-and-Master: invasives have higher fitness in all environments, but the advantage is most obvious in favorable environments (Fig.1c).

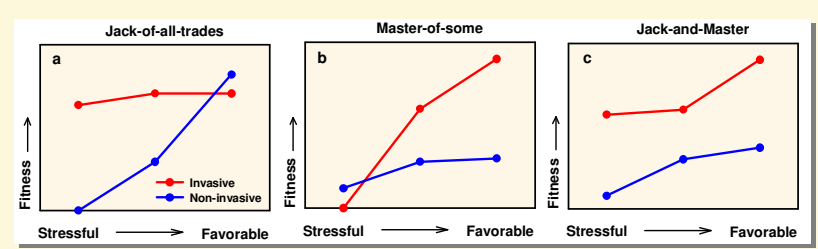

Fig. 1 Three possible phenotypic plasticity strategies for invasive species (red) and non-invasive species (blue). Redrawn from Richards et al (2006).

OBJECTIVE: To test the hypothesis that invasive and non-invasive species differ in their phenotypic plasticity.

TEST SPECIES: Three non-native dandelion species: Taraxacum officinale (TOF) - a widespread weed (Fig. 2).

T. laevigatum (TLA) - relatively rare in Ohio (Fig. 2).

T. kok-saghyz (TKS) - a potential crop species. A secondary objective was to assess the invasive potential of TKS using comparison results from TOF and TLA.

Fig. 2 Morphology of the three dandelions species and their distribution in Ohio: (left to right) the TLA, and potential crop TKS

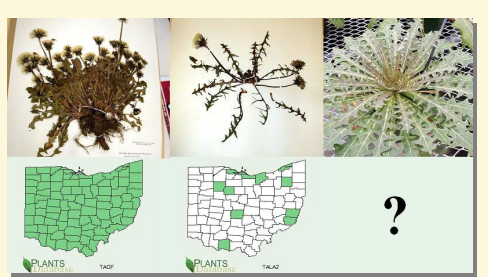

\section{Materials and Methods}

TOF and TLA seeds were collected from various locations in Columbus and Wooster, OH. TKS seeds were obtained from research materials at Ohio Agricultural Research \& Development Center. - The three species were compared in contrasting environments in

1 Germination: temperature (alternating or constant), light condition (light vs. dark), water potential gradient.

2 Greenhouse: light (full light vs. green shade) $\times$ soil moisture (dry vs. wet) $\times$ soil fertility (high vs. low)

3 Field: Competition (tall grass, short grass, no-grass) $\times$ soil fertility (high vs. low)

\section{Results}

Germination Experiment
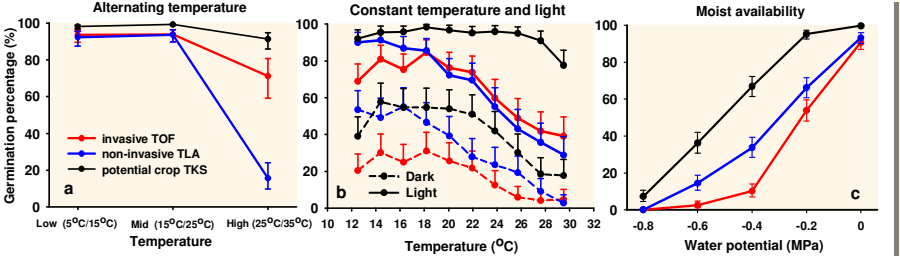

Stressful (high):

TOF > TLA, TKS: best

Stressful (dark):

Favorable (low and mid):
TOF $=$ TLA, TKS: best

TOF < TLA, TKS:

Favorable (light):

Stressful (dry): Jack-of-all-trades Favorable (moist):

OF = TLA, TKS: bes

Fig. 3 Germination patterns of the three dandelions species in different temperature (a and b), light (b) and water potential (c) environments. Error bar shows one standard error.

Greenhouse Experiment

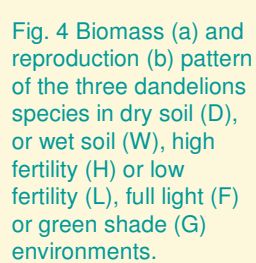

envionments.

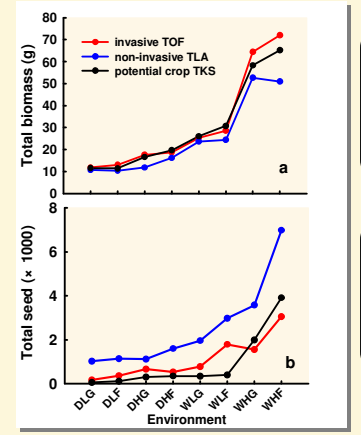

Biomass:

Slressful (dry, low fertility, shade): Favorable (wet, high fertility, full light) A. TKS TOF Master-of-some

\section{Reproduction:}

Stressful (dry, low fertilly,
TOF $=$ TLA, TKS $\sim$ TOF

STEFora,

TOF $<$ TLA, TKS TOF

Field Experiment
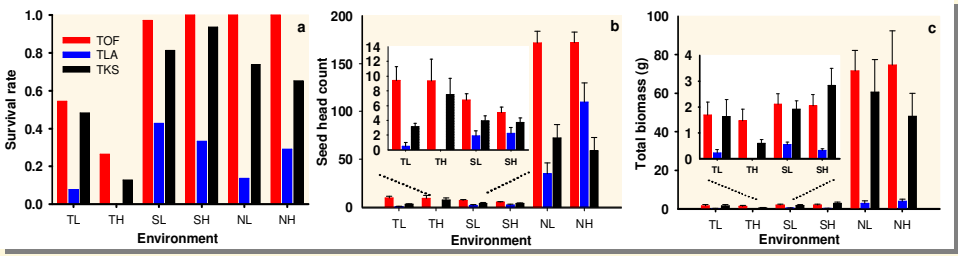

Survival:

Stressful (tall grass, low fertility):
TOF $>$ TLA, TKS TOF

Favorable (no grass, high fertilly):

TOF > TLA, TKS TOF

Jack-and-Master

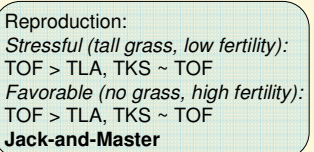

Biomass:

Stressful (tall grass, low fertility)
TOF $>$ TLA, TKS TOF

Favorable (no grass, high fertility)
TOF $>$ TLA, TKS $\sim$ TOF Jack-and-Master

Figure 5. Survival (a), reproduction (b) and biomass (c) of the three dandelions species different competitor (tall-, short-, or no-grass) and soil fertility (high or low) environments. Error bar shows one standard error.

\section{Conclusions}

The invasive TOF does differ from the non-invasive TLA in

phenotypic plasticity responses to

All three strategies (Jack-of-alltrades, Master-of-some, and Jackand-Master) were identified in different fitness components

(germination, growth, and

reproduction, Figs. $3,4,5)$.

- The non-invasive TLA exhibited

higher fitness under some

conditions in germination and

greenhouse experiments (Figs. 3

and 4).

The success of TOF might be attributed to:

- Germination only in favorable environments (light, moist)

- Higher biomass accumulation under high resource conditions

- High survival in all habitats

- Less reliance on seed reproduction

TKS performed more similar to TOF than to TLA, which suggests that it has some potential to

behave as a weed.

\section{Reference}

Richards, C. L., O. Bossdorf, N. Z. Muth, J. Gurevitch, and M. Pigliucci. 2006. Jack of all trades, master of some? On the role of phenotypic Letters 9.981-993

\section{Acknowledgment}

We thank David Tay, Kent Harrison, Emilie Regnier, and Maria Miriti for comments and suggestions, Steven St Martin for statistical advice, and numerous lab, greenhouse and field staff for their assistance.

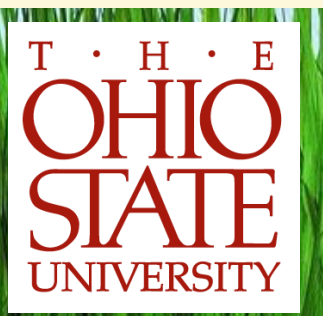

